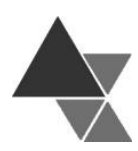

\title{
Padrões de consumo alimentar baseados no orçamento das famílias na região metropolitana de São Paulo
}

\author{
Davi Augusto Camargo ${ }^{1}$ e Luiz Fernando Satolo ${ }^{2}$
}

Os padrões alimentares são similaridades no consumo de alimentos que podem ser influenciadas pelo orçamento das famílias. A aplicação da Análise Fatorial Exploratória aos microdados da Pesquisa de Orçamentos Familiares - POF 2008/2009 evidencia essa relação. Na região metropolitana de São Paulo, considerando 16 subgrupos de alimentos e 4 níveis de renda, observa-se que a quantidade de alimentos adquirida para consumo no domicílio tende a ser maior em famílias com maior poder aquisitivo. Por outro lado, a porcentagem da variância total do consumo de alimentos explicada pela análise fatorial diminui à medida que a renda aumenta. Além disso, o padrão de consumo mais importante é distinto em cada nível de renda considerado. No nível de renda A e B, o principal padrão de consumo é voltado para alimentos saudáveis e de origem vegetal. No nível de renda $C$, o padrão de consumo mais importante não envolve expressiva parcela dos alimentos de origem vegetal. No nível de renda $\mathrm{D}$, o principal padrão de consumo é composto por alimentos de origem animal. Já, no nível de renda E, o padrão mais importante contém produtos processados e de preparação fácil e rápida.

Palavras-chave: alimento, consumo, padrão, renda.

\section{Patterns of food consumption based on household budgets in the metropolitan region of Sao Paulo}

Dietary patterns are similarities in food consumption that may be influenced by household budgets. The Exploratory Factor Analysis applied to IBGE's Pesquisa de Orçamentos Familiares - POF 2008/2009 microdata shows this relationship. In the metropolitan region of Sao Paulo, taking into account 16 food subgroups and 4 income levels, it can be observed that the amount of food acquired for household consumption tends to be higher in families with higher purchase power. On the other hand, the proportion of the total variance of food consumption explained by the factor analysis decreases with the increasing income. Moreover, the most important pattern of food consumption is different for each income level considered. In the income level A and B, the main pattern of consumption is towards healthy and plant foods. In the income level C, the most important consumption pattern does not comprise much of the food of plant origin. In the income level $\mathrm{D}$, the main pattern of consumption is based on animal products. Yet, in the income level E, the most important pattern includes processed and convenience food.

Keywords: food, consumption, pattern, income.

1 Bacharel em Ciências dos Alimentos, ESALQ/USP. Endereço para correspondência: Rua Gomes Carneiro no 545, Piracicaba, SP, Brasil. Telefone: (19)98376-1868. E-maik. davi.camargo@usp.br

2 Professor do Departamento de Economia, Administração e Sociologia, ESALQ/USP. Endereço para correspondência: Av. Pádua Dias no 11, Piracicaba, SP, Brasil, CEP: 13418-900. Telefone: (19)3429-4100.E-mail:1fsatolo@usp.br 


\section{INTRODUÇÃO E OBJETIVOS}

Os padrões de consumo alimentar são similaridades buscadas no consumo de alimentos pelo indivíduo ou pela sociedade. Tais similaridades incluem a dependência de um alimento com o outro, ou seja, o consumo de um alimento ocorre principalmente quando o outro também é consumido. Outra similaridade é a influência da renda sobre o consumo de determinado alimento, possibilitando que uma maior quantidade seja consumida quando a renda aumenta.

Os níveis de renda das familias podem estar relacionados aos padrões de consumo de diferentes formas, o que pode mudar de região para região e também com o decorrer do tempo. Tais padrões podem, por exemplo, indicar uma tendência à escolha por alimentos saudáveis, por alimentos industrializados ou por alimentos de origem vegetal ou animal.

A busca por padrões de consumo alimentar pode auxiliar o desenvolvimento das pesquisas de mercado, fornecendo uma base sólida inicial para analisar como o mercado de alimentos e sua economia funcionam. Conhecendo esses padrões, a indústria pode ofertar determinados alimentos conforme o grupamento das pessoas, conforme o estrato de rendimentos.

De forma geral, esse trabalho buscou avaliar os dados fornecidos pela Pesquisa de Orçamentos Familiares (POF) 2008-2009[1] referentes às famílias da região metropolitana de São Paulo - RMSP. Por meio da Análise Fatorial Exploratória, os dados foram comparados, pesquisando por padrões de consumo alimentar baseados no orçamento. Apenas os subgrupos de alimentos foram selecionados para a pesquisa.

Os padrões de consumo alimentar tendem a ser associados à renda das famílias, pois julga-se que quanto maior for a renda, maior será o consumo de determinado alimento. Entretanto, dependendo das preferências dos consumidores, isso pode não ser observado em todos os subgrupos de alimentos.

Além disso, quanto maior o orçamento, maior será a abrangência da escolha alimentar. Com uma grande variedade de produtos, indaga-se, por exemplo, se a escolha alimentar das famílias será mais saudável à medida que a renda aumenta. Ou então, se uma renda elevada pode estar associada à obesidade devido a um padrão de consumo de alimentos processados e altamente calóricos.

O tempo para se alimentar dentro do domicílio é escasso para a maioria das familias. E isso pode não apenas limitar os alimentos que são consumidos dentro do domicílio, mas também criar padrões de consumo distintos daqueles que são observados quando a alimentação é realizada fora dele. Tendo isso em vista, o presente trabalho busca relacionar a renda das famílias e os padrões alimentares, considerando apenas a alimentação dentro do domicilio.

A Tabela 1 apresenta a evolução da aquisição monetária domiciliar, variável que será utilizada para representar o consumo, por grupo de alimento, entre 1987 e 2008. 
Tabela 1. Aquisição (monetária) alimentar domiciliar per capita anual (em kilograma) por grupos,1987-2008

\begin{tabular}{|c|c|c|c|c|c|}
\hline Grupos & 1987 & 1995 & 2002 & 2008 & $\begin{array}{c}\Delta \\
(\%)\end{array}$ \\
\hline 1. Cereais e leguminosas & 47,0 & 33,6 & 28,5 & 30,0 & -36 \\
\hline 2. Hortaliças & 46,7 & 28,1 & 25,3 & 26,1 & -44 \\
\hline 3. Frutas & 59,7 & 44,6 & 28,0 & 36,3 & -39 \\
\hline 4. Cocos, castanhas e nozes & 0,3 & 0,2 & 0,3 & 0,2 & -22 \\
\hline 5. Farinhas, féculas e massas & 13,7 & 9,0 & 8,2 & 8,2 & -40 \\
\hline 6. Panificados & 28,5 & 26,6 & 23,8 & 26,1 & -8 \\
\hline 7. Carnes & 26,1 & 30,7 & 22,0 & 22,7 & -13 \\
\hline 8. Vísceras & 1,1 & 0,8 & 0,8 & 0,4 & -67 \\
\hline 9. Pescados & 2,9 & 2,5 & 1,9 & 1,6 & -45 \\
\hline 10. Aves e ovos & 24,1 & 20,8 & 12,4 & 13,3 & -45 \\
\hline 11. Laticínios & 87,1 & 77,4 & 51,3 & 51,5 & -41 \\
\hline 12. Açúcares, doces e produtos de confeitaria & 24,8 & 16,8 & 14,4 & 15,9 & -36 \\
\hline 13. Sais e condimentos & 7,8 & 4,9 & 5,5 & 5,7 & -27 \\
\hline 14. Óleos e gorduras & 13,3 & 8,0 & 7,8 & 7,8 & -41 \\
\hline 15. Bebidas e infusões & 27,9 & 36,8 & 53,8 & 56,4 & 102 \\
\hline 16. Alimentos preparados e misturas industriais & 2,6 & 5,5 & 4,3 & 5,6 & 116 \\
\hline
\end{tabular}

Fonte: adaptado de IBGE (2010).

É interessante observar que, de maneira geral, houve uma diminuição no consumo da maior parte dos grupos de alimentos dentro do domicílio, o que pode estar associado a um aumento da participação da alimentação fora do domicílio. Além disso, algumas alterações no padrão de consumo alimentar do brasileiro dentro do domicílio já podem ser observadas ao longo do tempo. Por exemplo, enquanto o consumo de vísceras caiu $67 \%$ de 1987 para 2008, o consumo de panificados caiu apenas $8 \%$ e o consumo de alimentos preparados cresceu $116 \%$.

Segundo Moratoya et al.[2], a urbanização, a globalização e a renda estão entre os fatores que alteram os padrões de consumo. O consumo saudável de alimentos é preferencialmente adotado por grupos com rendimentos mais elevados. Ainda que a globalização e a urbanização cooperem para a disseminação de uma ampla variedade de produtos alimentares para a sociedade, isso não implica em escolhas alimentares mais saudáveis.

Segundo Newby e Tucker ${ }^{[3]}$, padrões alimentares podem ser identificados a priori ou a posteriori. Nos padrões alimentares a priori, são propostos índices que são avaliados de acordo com o que se entende por nutrição saudável ou outras recomendações propostas. Segundo Olinto[4], tais índices informam, por exemplo, a totalização de alimentos ou a totalização de nutrientes.

Quanto à definição dos padrões alimentares a posteriori, os dados são obtidos com base empírica e sustentados com base em análise estatística. Os modelos alimentares são associados com diferentes biomarcadores, sendo avaliados pela análise fatorial ou por clustering. Ainda que ocorra alguma diferença na aproximação e interpretação, há algumas evidências que modelos alimentares subjacentes são revelados por ambos métodos ${ }^{[2]}$.

Conforme Kourlaba e Panagiotakos [5], não há métodos diretos que permitam avaliar padrões alimentares. Uma das aproximações indiretas para determiná-los baseia-se na composição dos índices de qualidade da dieta usando o conhecimento atual de nutrição, chamada de modelo de padrão alimentar teoricamente definido. A outra tem base em técnicas estatísticas como análise do componente principal e o clustering.

As aproximações indiretas para avaliar os padrões alimentares alicerçam os estudos sobre o assunto. Os diferentes tipos de aproximação permitem que um amplo espectro de novas análises seja realizado, com o auxílio das técnicas estatísticas ou dos conhecimentos de nutrição[5]. 
O padrão alimentar revela a complexidade existente na alimentação, uma vez que não há o consumo isolado de alimentos ou nutrientes. De acordo com Olinto ${ }^{[4]}$, a aquisição de alimentos no cotidiano ocorre de maneira simples, podendo o indivíduo ter padrões alimentares diferentes, dada a grande diversidade de alimentos disponíveis.

No Brasil, como mostra Garcia[6], as tendências no consumo de alimentos convergem à homogeneidade, com aumento no consumo de produtos industrializados e diminuição no consumo de produtos básicos da alimentação.

Ainda que a padronização dos hábitos e práticas alimentares tenha sido pautada pelos programas de suplementação alimentar amparados pelos organismos internacionais, estes tinham objetivos ideológicos e econômicos bem estruturados. Segundo Pinheiro[7] o incentivo à produção e ao consumo local de alimentos básicos para o desenvolvimento sustentável, não era um modelo de desenvolvimento social apoiado, sendo de maior proveito que houvesse uma relação de dependência.

Do ponto de vista epidemiológico, Olinto[4] destaca que os padrões alimentares são os principais fatores de risco modificáveis para a prevenção de doenças. E, de acordo com Garcia ${ }^{[6]}$, o consumo de alimentos ditos invisíveis - como sal, açúcar e óleo é determinado pela condição socioeconômica da população.

Quanto às mudanças estruturais na alimentação, Garcia[6] enfatiza que ocorre influência da renda e do tempo nas escolhas alimentares. $O$ estilo de vida (hábitos e trabalho) dos indivíduos pode trazer mudanças significativas para a disponibilidade de tempo e de como será distribuída a renda na escolha dos alimentos. Além disso, há dificuldade na adoção de novas práticas visando uma alimentação saudável, possibilitando um entendimento de que a mudança ou a inserção de novos hábitos e novas estruturas alimentares não ocorre de maneira expressivamente simples ou fácil.
De acordo com Hoffmann ${ }^{[8]}$, no Brasil, cerca de $40,2 \%$ das pessoas comem fora do domicilio, enquanto que $59,8 \%$ se alimentam no domicilio. Esses números revelam que as pessoas procuram otimizar o seu tempo, mas, ao contrário do que se pode pensar, essa prática não é adotada pela maioria da população. A alimentação no domićlilo tem uma associação com a renda familiar, sendo que normalmente as famílias de renda mais baixa possuem o hábito de fazer as refeições em casa.

Hoffmann ${ }^{[8]}$ relata que o grupo de pessoas que se alimenta fora de casa, além de possuir maior poder aquisitivo e maior escolaridade, também é mais jovem em relação ao grupo das pessoas que se alimentam no domicilio ( 31,2 versus 40,5 anos). Outra associação entre os fatores é que os indivíduos que se alimentam fora do domicilio possuem maior ingestão calórica $(2.064 \mathrm{kcal})$ quando comparados aos indivíduos que se alimentam dentro do domicilio (1.801 kcal).

Essa última constatação, a diferença na ingestão calórica, talvez seja influenciada pela maior liberdade desses indivíduos no momento da escolha e compra dos alimentos. No domicílio, é comum que a diversidade de alimentos seja menor, não havendo muita variação na sua aquisição, seja pela disponibilidade local ou pela falta de conhecimento no preparo de distintos alimentos. Fora do domicilio, entretanto, o cenário para alimentação é complemente diferente, visto que o indivíduo é livre para adquirir praticamente qualquer alimento, a qualquer momento do seu dia.

\section{MATERIAL E MÉTODOS}

Os dados analisados correspondem às quantidades médias (em kg) adquiridas, pelas famílias da região metropolitana de São Paulo (consumo dentro do domicílio) disponibilizados pela POF 2008-2009[1]. Os subgrupos de alimentos considerados estão listados na Tabela 2. 
Tabela 2. Informações relativas aos subgrupos e siglas correspondentes

\begin{tabular}{lc}
\hline \multicolumn{1}{c|}{ Subgrupo } & Abreviatura \\
\hline Açúcares e Derivados & $\mathrm{AD}$ \\
Aves e Ovos & $\mathrm{AO}$ \\
Alimentos Preparados & $\mathrm{AP}$ \\
Bebidas e Infusões & $\mathrm{BI}$ \\
Cereais, Legumes e Oleaginosas & $\mathrm{CLO}$ \\
Carnes, Vísceras e Pescados & $\mathrm{CVP}$ \\
Enlatados e Conserva & $\mathrm{EC}$ \\
Frutas & $\mathrm{F}$ \\
Farinhas, Féculas e Massas & FFM \\
Leite e Derivados & $\mathrm{LD}$ \\
Legumes e Verduras & LV \\
Outros (Alimentação no Domicílio) & OAND \\
Óleos e Gorduras & OG \\
Panificados & P \\
Sal e Condimentos & SC \\
Tubérculos e Raízes & TR \\
\hline
\end{tabular}

Fonte: elaborado pelos autores.

Os microdados da POF 2008-2009 foram separados em quatro níveis de renda, definidos com base na classificação utilizada pelo Centro de Políticas Sociais - CPS ${ }^{[9]}$ em 2008:

- classe E: renda familiar per capita menor que $\mathrm{R} \$$ 768,00 ;

- classe D: renda familiar per capita maior ou igual a $\mathrm{R} \$ 768,00$ e menor que $\mathrm{R} \$ 1.064,00$;

- classe C (média): renda familiar per capita maior ou igual a $\mathrm{R} \$ 1.064,00$ e menor que $\mathrm{R} \$ 4.591,00$;

- classe A e B (elite): renda familiar per capita maior ou igual a $\mathrm{R} \$ 4.591,00$.

Esse tratamento foi realizado com a finalidade de facilitar a análise e compreensão dos resultados, uma vez que os padrões de consumo podem então ser calculados e avaliados separadamente dentro de cada classe de renda.

É importante ressaltar que os microdados da POF não constituem uma amostra aleatória simples e, por isso, não devem ser analisados diretamente. Cada domicílio possui um fator de expansão que representa a sua participação na população total, o qual foi utilizado para ponderar as observações utilizadas na análise fatorial. Assim, a amostra de 272 domicílios das classes A e B equivale a uma população de aproximadamente 1,4 milhões de domicílios, a amostra de 763 domicílios da classe C, corresponde a uma população de cerca de 3,6 milhões de domicílios, a amostra de 98 domicílios da classe $\mathrm{D}$ equivale a uma população de mais de 500 mil domicílios e a amostra de 81 domicílios da classe E corresponde a uma população de praticamente 400 mil domicílios.

Os padrões de consumo alimentar foram extraídos por Análise Fatorial Exploratória - AFE por meio do programa IBM SPSS Statistics 22.

A AFE é uma técnica de análise multivariada que tem por objetivo revelar estruturas latentes (fatores) que explicam a covariância entre os itens analisados ${ }^{[10]}$. Na literatura específica sobre o tema, essa tem sido uma das técnicas mais utilizadas para identificar os padrões alimentares a posterior [3,11,12].

Nos modelos de análise fatorial, cada um dos itens é representado como uma combinação linear de determinado número de fatores comuns e de um fator específico[13]. A aplicação da AFE pressupõe que a matriz de correlações entre as variáveis seja passível de fatoração, o que pode ser avaliado por meio do critério de Kaiser-Meyer-Olkin (índice 
KMO, também conhecido como índice de adequação da amostra) e pelo teste de esfericidade de Bartlett[10].

O método de extração utilizado foi a Fatoração de Eixo Principal, apontado na literatura como o que apresentada melhores resultados quando os dados apresentam distribuição não-normal. $\mathrm{O}$ número de fatores retidos na AFE foi definido com base no critério de Kaiser-Guttman (autovalor >1). Como os subgrupos de alimentos podem, teoricamente, integrar mais de um padrão de consumo, optou-se por uma rotação oblíqua dos fatores (especificamente, foi utilizado o método Oblimin direito com delta 0$)^{[10]}$.

\section{RESULTADOS E DISCUSSÃO}

A Tabela 3 apresenta a média, o desvio padrão e o número de observações para cada um dos quatro níveis de renda selecionados.

De forma geral, como era de se esperar, a aquisição média em cada subgrupo de alimentos tende a ser maior nos níveis de renda mais elevados. Mas há algumas exceções, a saber:

- o consumo de cereais, leguminosas e oleaginosas (CLO) nas classes A e B (elite) é inferior às médias observadas nas classes C (média) e D;

- o consumo de óleos e gorduras (OG) na classe C é inferior à média observada na classe $\mathrm{D}$;

- o consumo de outros alimentos (OAND) na classe $\mathrm{D}$ é inferior à média observada na classe $\mathrm{E}$.

Além disso, observa-se que o consumo tende a ser mais heterogêneo nos níveis de renda mais elevados (atentar para os valores de desvios padrão obtidos quando são analisados os dados das classes com maiores rendimentos). Nesse caso, as exceções são:

- o consumo de cereais, leguminosas e oleaginosas (CLO) nas classes A e B (elite) é mais homogêneo que na classe $\mathrm{C}$ (média) e na classe $\mathrm{D}$;
- o consumo de farinhas, féculas e massas (FFM), açúcares e derivados (AD) e bebidas e infusões (BI) na classe $C$ são mais homogêneos que na classe D;

- o consumo de aves e ovos (AO) e outros alimentos (OAND) na classe $\mathrm{D}$ são mais homogêneos que na classe $\mathrm{E}$.

Combinadas, essas estatísticas descritivas indicam que o consumo de alimentos tende a ser menor e mais homogêneo nas classes mais baixas, D e E. Isso ocorre porque, com orçamentos menores, as escolhas dessas famílias são limitadas, tanto em quantidade quanto em qualidade dos produtos a serem adquiridos. Por outro lado, nas classes de renda mais alta, os resultados corroboram a ideia de que os consumidores possuem maior liberdade para exercer suas preferências individuais de consumo alimentar, o que se reflete na maior heterogeneidade das observações.

As correlações dos consumos nos subgrupos também apresentaram diferenças entre as classes de renda, variando: de $-0,007$ entre CLO e AP até $+0,602$ entre LV e F nas classes A e B; de -0,005 entre $\mathrm{AP}$ e $\mathrm{EC}$ até +0,588 entre LV e F na classe C; de 0,006 entre $\mathrm{F}$ e CVP até $+0,859$ entre TR e LV na classe D; de -0,097 entre CVP e OAND até +0,775 entre LD e SC na classe E.

A estatística $\mathrm{KMO}$ e os resultados do teste de Bartlet são apresentados na Tabela 4. Como os valores de KMO são próximos de 0,8 para todos os níveis de renda, os dados são adequados à aplicação da Análise Fatorial Exploratória. Esses resultados são corroborados pelo teste de Bartlet (rejeição da hipótese nula - variáveis analisadas não são correlacionadas ao nível de significância de 1). 
Tabela 3. Estatísticas descritivas

\begin{tabular}{|c|c|c|c|c|c|c|c|c|}
\hline \multirow{2}{*}{ Subgrupo } & \multicolumn{2}{|c|}{ Classe A e B } & \multicolumn{2}{|c|}{ Classe C } & \multicolumn{2}{|c|}{ Classe D } & \multicolumn{2}{|c|}{ Classe E } \\
\hline & Média & Desvio Padrão & Média & Desvio Padrão & Média & Desvio Padrão & Média & Desvio Padrão \\
\hline CLO & 161,3 & 341,3 & 226,5 & 551,2 & 194,1 & 385,3 & 67,5 & 215,1 \\
\hline FFM & 133,5 & 275,3 & 94,8 & 219,0 & 73,7 & 244,9 & 37,4 & 124,3 \\
\hline TR & 67,7 & 125,2 & 44,3 & 95,7 & 36,5 & 87,6 & 8,6 & 39,0 \\
\hline $\mathrm{AD}$ & 271,8 & 608,0 & 113,6 & 241,9 & 90,9 & 252,8 & 21,3 & 87,5 \\
\hline LV & 178,2 & 280,7 & 104,3 & 179,0 & 91,7 & 171,4 & 27,0 & 58,7 \\
\hline $\mathrm{F}$ & 334,1 & 469,7 & 163,7 & 305,7 & 85,0 & 157,8 & 36,6 & 85,2 \\
\hline CVP & 925,2 & $1.205,6$ & 678,0 & $1.103,7$ & 459,2 & 581,5 & 226,0 & 421,4 \\
\hline $\mathrm{AO}$ & 250,8 & 487,3 & 174,3 & 316,3 & 133,9 & 222,7 & 128,9 & 259,3 \\
\hline LD & 686,2 & 863,8 & 416,9 & 611,9 & 303,4 & 419,7 & 141,7 & 310,8 \\
\hline $\mathrm{P}$ & 615,3 & 725,4 & 425,7 & 513,9 & 287,9 & 321,1 & 145,6 & 189,1 \\
\hline OG & 115,1 & 298,9 & 53,7 & 158,1 & 58,0 & 157,3 & 16,0 & 49,7 \\
\hline $\mathrm{BI}$ & 632,2 & $1.024,1$ & 277,4 & 427,4 & 245,6 & 432,1 & 64,4 & 122,5 \\
\hline $\mathrm{EC}$ & 67,7 & 203,8 & 30,2 & 128,3 & 23,2 & 111,1 & 2,1 & 19,2 \\
\hline SC & 120,0 & 270,2 & 83,4 & 189,2 & 49,8 & 141,7 & 31,9 & 110,7 \\
\hline $\mathrm{AP}$ & 322,7 & 781,7 & 133,4 & 392,4 & 53,7 & 257,3 & 49,1 & 227,0 \\
\hline OAND & 600,4 & $2.342,8$ & 343,9 & $2.262,8$ & 29,7 & 139,0 & 267,0 & $1.190,0$ \\
\hline Observações & \multicolumn{2}{|c|}{1.392 .434} & \multicolumn{2}{|r|}{ 3.575.202 } & \multicolumn{2}{|r|}{504.739} & \multicolumn{2}{|r|}{394.238} \\
\hline
\end{tabular}

Fonte: resultados da pesquisa.

Tabela 4. Índice KMO e teste de Bartlett

\begin{tabular}{c|c|c|c}
\hline Nível de renda & $\begin{array}{c}\text { Índice KMO de adequação } \\
\text { da amostra }\end{array}$ & $\begin{array}{c}\text { Teste de esfericidade de } \\
\text { Bartlett }\end{array}$ & $\begin{array}{c}\text { Probabilidade de } \\
\text { significância }\end{array}$ \\
\hline A e B & 0,799 & 5.813 .798 & 0,000 \\
C & 0,859 & 16.162 .374 & 0,000 \\
D & 0,799 & 5.486 .372 & 0,000 \\
E & 0,759 & 4.614 .775 & 0,000 \\
\hline
\end{tabular}

Fonte: resultados da pesquisa.

Entretanto, o número de fatores que apresentaram autovalor maior que 1 variou entre os níveis de renda, levando à retenção de um número diferente de fatores para cada classe. No caso da categoira elite, foram retidos 5 fatores, ou seja, foram identificados 5 padrões de consumo. Para a classe média, esse número caiu para apenas 3 . Nas classes de renda mais baixa, $\mathrm{D}$ e $\mathrm{E}$, foram identificados 4 padrões de consumo de alimentos em cada uma das categorias.

$\mathrm{Na}$ Tabela 5, pode-se observar que a proporção da variância de cada subgrupo de alimento explicada pelos fatores extraídos na análise, conhecida pelo nome de comunalidade, também difere entre os níveis de renda. Como referência, de acordo com a literatura especializada, considera-se que variáveis que apresentam valores de comunalidade menor que 0,5 não podem ser explicadas pela análise fatorial elaborada. Dos 16 subgrupos analisados, isso aconteceu em 10 deles, para o nível de renda A e B, 12 para o nível de renda C, 7 para o nível de renda $\mathrm{D}$ e apenas 3 para o nível de renda $\mathrm{E}$.

Consequentemente, conforme evidenciado na Tabela 6 , a porcentagem da variância total explicada pela análise fatorial é maior nos níveis de renda mais baixos. Tomando-se, por exemplo, os três fatores mais importantes, observa-se que o percentual acumulado da variância total explicada aumenta à medida que o nível de renda diminui. Essa constatação reafirma a ideia de que o padrão de consumo tende a ser mais homogêneo quando o nível de renda é mais baixo. Entretanto, cabe ressaltar que, como foi utilizado um método oblíquo de rotação, pode existir correlação entre os fatores (o que levaria a uma superestimação da variância acumulada explicada).

A Tabela 7 apresenta os subgrupos de alimentos com carga fatorial maior que 0,2 ou menor que $-0,2$ em cada fator extraído para os diferentes níveis 
de renda. Esses valores identificam as variáveis mais importantes para cada fator, ou seja, para cada padrão de consumo.

Observa-se que o padrão de consumo mais importante no nível de renda mais elevado (A e B) é vegetariano, pois carnes, vísceras e pescados $(\mathrm{CVP})$ e aves e ovos (AO) não estão entre as variáveis mais importantes do Fator 1. O segundo padrão de consumo mais importante nesse nível de renda (Fator 2), composto por tubérculos e raízes (TR), legumes e verduras $(\mathrm{LV})$, frutas $(\mathrm{F})$ e aves e ovos $(\mathrm{AO})$, pode ser associado a um estilo de vida mais saudável e fitness. $\mathrm{O}$ terceiro padrão de consumo mais relevante (Fator 3), ao contrário, envolve alimentos processados e ricos em carboidratos simples. $\mathrm{O}$ quarto padrão de consumo mais importante (fator 4) é composto principalmente por carnes vermelhas e brancas (CVP e AO), tipicamente adquiridas em conjunto para churrasco. $\mathrm{O}$ último padrão de consumo identificado para o nível de renda A e B (Fator 5) é composto por AO e LD, dois subgrupos que tem compostos por proteína animal que normalmente são consumidos em substituição à carne vermelha.

Na classe média, C, o padrão de consumo mais importante (Fator 1) não compreende grande parte dos alimentos de origem vegetal (LV, TR e F), podendo ser associado à procura por conforto e praticidade, já que inclui grupos como $\mathrm{AD}, \mathrm{BI}$ e $\mathrm{EC}$, ao mesmo tempo apresenta os grupos FFM e CLO. O segundo padrão de consumo mais importante (Fator 2) pode ser associado à procura por um estilo de vida mais saudável, e o terceiro (Fator 3) a alimentos processados e mais calóricos, como os encontrados em redes de fast food. Entretanto, ao contrário do grupo identificado como elite, o principal padrão de consumo não é vegetariano, pois inclui CVP.

Tabela 5. Comunalidades

\begin{tabular}{c|c|c|cc}
\hline Subgrupo & Nível de renda A e B & Nível de renda C & Nível de renda D & Nível de renda E \\
\hline CLO & 0,337 & 0,403 & 0,581 & 0,810 \\
FFV & 0,352 & 0,484 & 0,803 & 0,772 \\
TR & 0,493 & 0,348 & 0,861 & 0,668 \\
AD & 0,359 & 0,471 & 0,949 & 0,816 \\
LV & 0,675 & 0,796 & 0,807 & 0,578 \\
F & 0,536 & 0,430 & 0,431 & 0,749 \\
CVP & 0,547 & 0,291 & 0,311 & 0,716 \\
AO & 0,488 & 0,187 & 0,369 & 0,254 \\
LD & 0,442 & 0,574 & 0,216 & 0,661 \\
P & 0,636 & 0,348 & 0,493 & 0,547 \\
OG & 0,605 & 0,593 & 0,476 & 0,809 \\
BI & 0,288 & 0,360 & 0,675 & 0,484 \\
EC & 0,323 & 0,170 & 0,736 & 0,909 \\
SC & 0,686 & 0,627 & 0,698 & 0,838 \\
AP & 0,114 & 0,045 & 0,135 & 0,576 \\
OAND & 0,026 & 0,010 & & 0,017 \\
\hline
\end{tabular}

Fonte: resultados da pesquisa.

Tabela 6. Variância total explicada (\% acumulado)

\begin{tabular}{c|c|c|c|c}
\hline Fator & Nível de renda A e B & Nível de renda C & Nível de renda D & Nível de renda E \\
\hline 1 & 24,43 & 26,64 & 39,80 & 37,39 \\
2 & 31,78 & 35,25 & 48,04 & 49,16 \\
3 & 37,25 & 38,36 & 54,15 & 58,21 \\
4 & 40,49 & - & 57,86 & 63,77 \\
5 & 43,17 & - & - & - \\
\hline
\end{tabular}

Fonte: resultados da pesquisa. 
O principal padrão de consumo do nível de renda D (Fator 1) exclui grupos de alimentos não processados como F, LV e TR, e, concomitantemente, apresenta os grupos de alimentos de origem animal (CVP, AO e LD), sendo, portanto, um padrão de consumo baseado em produtos de origem animal. No Fator 2, os grupos TR e LV não foram importantes na participação do padrão de consumo. O Fator 3 teve os grupos AP e AD como os menos importantes, sendo $\mathrm{SC}$ e $\mathrm{P}$ os dois grupos mais elevados neste fator, indicando a importância de especiarias e massas. $\mathrm{O}$ Fator 4 teve o FFM como o grupo menos importante, enquanto que $\mathrm{LD}$ e $\mathrm{P}$, ganharam maior destaque.

Já no nível de renda E, o principal padrão de consumo (Fator 1) foi composto principalmente pelos subgrupos $\mathrm{AD}$ e $\mathrm{EC}$, que pode indicar a procura por alimentos de fácil preparo e de consumo rápido. $\mathrm{O}$ grupo $\mathrm{AD}$ compreende todos os derivados do açúcar e o próprio açúcar, mostrando que essa é uma das principais fontes de energia utilizadas nas classes mais baixas. Para o Fator 2, o grupo mais importante foi CLO, que compreende arroz e feijão, base alimentar do nível de renda E. Alimentos preparados e de origem animal são os mais importantes para o Fator 3, revelando um estilo de vida que engloba grande consumo de calorias e de proteínas animais. Ainda, há a participação de grupos como LD e $\mathrm{P}$, que englobam grande quantidade de proteínas e gorduras (LD) e de carboidratos simples ou complexos $(\mathrm{P})$. O subgrupo $\mathrm{F}$ foi o mais importante para o Fator 4, sendo o subgrupo LV o menos importante para este fator.

Tabela 7. Matriz de padrão

\begin{tabular}{|c|c|c|c|c|c|c|c|c|c|c|c|c|c|c|c|c|}
\hline \multirow[b]{2}{*}{ Subgrupo } & \multicolumn{5}{|c|}{ Nível de renda A e B } & \multicolumn{3}{|c|}{ Nível de renda $\mathrm{C}$} & \multicolumn{4}{|c|}{ Nível de renda D } & \multicolumn{4}{|c|}{ Nível de renda $\mathrm{E}$} \\
\hline & $\begin{array}{c}\text { Fator } \\
1\end{array}$ & $\begin{array}{c}\text { Fator } \\
2 \\
\end{array}$ & $\begin{array}{c}\text { Fator } \\
3 \\
\end{array}$ & $\begin{array}{c}\text { Fator } \\
4 \\
\end{array}$ & $\begin{array}{c}\text { Fator } \\
5\end{array}$ & $\begin{array}{c}\text { Fator } \\
1\end{array}$ & $\begin{array}{c}\text { Fator } \\
2 \\
\end{array}$ & $\begin{array}{c}\text { Fator } \\
3 \\
\end{array}$ & $\begin{array}{c}\text { Fator } \\
1 \\
\end{array}$ & $\begin{array}{c}\text { Fator } \\
2\end{array}$ & $\begin{array}{c}\text { Fator } \\
3 \\
\end{array}$ & $\begin{array}{c}\text { Fator } \\
4 \\
\end{array}$ & $\begin{array}{c}\text { Fator } \\
1\end{array}$ & $\begin{array}{c}\text { Fator } \\
2 \\
\end{array}$ & $\begin{array}{c}\text { Fator } \\
3 \\
\end{array}$ & $\begin{array}{c}\text { Fator } \\
4 \\
\end{array}$ \\
\hline CLO & 0,357 & - & - & - & 0,284 & 0,666 & - & - & 0,720 & - & - & - & - & 0,888 & - & - \\
\hline FFV & - & - & - & - & 0,468 & 0,653 & - & - & 0,464 & - & - & 0,512 & 0,714 & 0,433 & - & - \\
\hline TR & 0,215 & 0,609 & - & - & - & - & 0,523 & - & - & 0,873 & - & - & 0,479 & 0,301 & 0,376 & 0,254 \\
\hline $\mathrm{AD}$ & 0,219 & - & 0,46 & - & - & 0,621 & - & - & 0,481 & - & 0,665 & - & 0,904 & - & - & - \\
\hline LV & - & 0,793 & - & - & - & - & 0,929 & - & - & 0,823 & - & - & - & - & 0,250 & 0,662 \\
\hline $\mathrm{F}$ & - & 0,747 & - & - & - & - & 0,692 & - & - & 0,71 & - & - & - & - & - & 0,839 \\
\hline CVP & - & - & - & 0,692 & - & 0,323 & 0,223 & - & 0,523 & - & - & - & - & 0,476 & 0,588 & - \\
\hline $\mathrm{AO}$ & - & 0,224 & - & 0,252 & 0,538 & 0,302 & 0,211 & - & 0,654 & - & - & - & 0,270 & - & 0,310 & - \\
\hline LD & 0,209 & - & 0,257 & - & 0,378 & 0,46 & - & 0,412 & 0,270 & - & - & 0,233 & 0,708 & - & 0,252 & - \\
\hline $\mathrm{P}$ & - & - & 0,739 & - & - & - & - & 0,474 & - & 0,394 & 0,265 & 0,253 & 0,621 & - & 0,234 & - \\
\hline OG & 0,683 & - & - & - & - & 0,813 & - & 0,252 & 0,546 & - & - & 0,413 & - & 0,763 & - & 0,430 \\
\hline BI & - & - & 0,454 & - & - & 0,481 & - & - & 0,526 & 0,267 & - & - & - & 0,249 & 0,419 & - \\
\hline EC & 0,374 & - & - & - & - & 0,429 & - & - & - & 0,383 & 0,316 & 0,437 & 0,971 & 0,253 & 0,228 & - \\
\hline SC & 0,808 & - & - & - & - & 0,752 & - & - & 0,404 & - & 0,247 & 0,441 & 0,763 & - & 0,205 & - \\
\hline $\mathrm{AP}$ & - & - & 0,314 & - & - & - & - & 0,223 & - & - & 0,885 & - & - & - & 0,722 & - \\
\hline OAND & - & - & - & - & - & - & - & - & - & - & - & 0,388 & - & - & - & - \\
\hline
\end{tabular}

Fonte: resultados de pesquisa.

\section{CONCLUSÃO}

O objetivo do trabalho foi analisar o padrão de consumo alimentar baseado no orçamento de familias da RMSP.

A matriz de padrão, que apresenta os fatores extraídos da análise, permitiu identificar os diferentes padrões de consumo subjacentes. Observou-se que tais fatores apresentam diferenças entre os níveis de renda, de tal forma que se pode determinar os principais padrões de consumo com base no orçamento das famílias da RMSP.
O trabalho possui limitações, pois apenas os subgrupos de alimentos foram analisados para cada nível de renda. Para trabalhos que necessitem identificar padrões mais precisos como exemplo: o consumo de Feijão-fradinho e Arroz integral comparando entre os níveis de renda A e C, procedimento semelhante pode ser utilizado para analisar dados desagregados do consumo. 


\section{AGRADECIMENTOS}

Os autores agradecem à Profa. Dra. Lilian M.

de L. Cunha pelas contribuições para o desenvolvimento da pesquisa.

\section{REFERÊNCIAS}

[1] IBGE - Instituto Brasileiro de Geografia e Estatística. Pesquisa de Orçamentos Familiares 2008-2009 - POF. Rio de Janeiro: IBGE; 2010.

[2] Moratoya EE, Carvalhaes GC, Wander AE, Almeida LMMC. Mudanças no padrão de consumo alimentar no Brasil e no mundo. Revista de Política Agrícola. 2013;22(1):72-84.

[3] Newby PK, Tucker KL. Empirically derived eating patterns using factor or cluster analysis: a review. Nutr. Rev. 2004;62(5):177-203.

[4] Olinto MTA. Padrões alimentares: análise de componentes principais. In: Kac G, Sichieri R, Gigante DP. Epidemiologia Nutricional. Rio de Janeiro: Editora Fiocruz/Atheneu; 2007. p. 213-225.

[5] Kourlaba G, Panagiotakos DB. Dietary quality indices and human health: a review. Maturitas. 2009;62(1):1-8.

[6] Garcia WDG. A comida, a dieta, o gosto: a mudança na cultura alimentar urbana [tese]. São Paulo: Universidade de São Paulo; 1999.

[7] Pinheiro ARO. Reflexões sobre o processo histórico/político de construção da lei orgânica de segurança alimentar e nutricional. Segur. Aliment. Nutr. 2008;15(2):115.

[8] Hoffmann R. Comparando a alimentação dentro e fora do domicílio, no Brasil, em 2008-2009. Segur. Aliment. Nutr. 2013;20(1):1-12.

[9] CPS - Centro de Políticas Sociais. A Nova Classe Média. Rio de Janeiro: FGV/IBRE; 2008.

[10] Damásio BF. Uso da análise fatorial exploratória em psicologia. Avaliação Psicológica. 2012;11(2):213-228.

[11] Borges CA, Rinalde AE, Conde WL, Mainardi GM, Behar D, Slater B. Padrões alimentares estimados por técnicas multivariadas: uma revisão da literatura sobre os procedimentos adotados nas etapas analíticas. Revista Brasileira de Epidemiologia. 2015;18(4):837-857.
[12] Carvalho CA, Fonsêca PCA, Nobre LN, Priore SE, Fransceschini SCC. Metodologias de identificação de padrões alimentares a posteriori em crianças brasileiras: revisão sistemática. Ciênc. Saúde Coletiva. 2016;21(1):143-154.

[13] Hoffmann R. Análise Estatística de Relações Lineares e Não-Lineares. São Paulo: LP Books; 2011. 